TRANSACTIONS OF THE

AMERICAN MATHEMATICAL SOCIETY

Volume 360, Number 11, November 2008, Pages 5869-5881

S 0002-9947(08)04482-6

Article electronically published on June 26, 2008

\title{
TOPOLOGICAL TRIVIALITY OF SMOOTHLY KNOTTED SURFACES IN 4-MANIFOLDS
}

\author{
HEE JUNG KIM AND DANIEL RUBERMAN
}

\begin{abstract}
Some generalizations of the Fintushel-Stern rim surgery are known to produce smoothly knotted surfaces. We show that if the fundamental groups of their complements are standard, then these surfaces are topologically unknotted.
\end{abstract}

\section{INTRODUCTION}

Fintushel and Stern described [6] a surgery operation on a torus $T$ embedded with trivial normal bundle in a smooth 4-manifold $X$, and used this construction to build many 4-manifolds with interesting properties. The construction depends on a choice of a knot $K$ in $S^{3}$, and the choice of a diffeomorphism $\varphi: \partial \nu(T) \rightarrow S^{1} \times \partial E(K)$ (subject to certain restrictions), and gives rise to a 4-manifold $X_{K}(\varphi)$. When this operation is applied to a torus embedded in a neighborhood of a surface $\Sigma$ in $X$ (and linking $\Sigma$ in a simple fashion), the new manifold $X_{K}(\varphi)$ is diffeomorphic to $X$. However, the construction, called rim surgery, produces a new surface $\Sigma_{K}(\varphi)$ in $X$ which Fintushel and Stern showed [5] may be smoothly nonisotopic to $\Sigma$.

In the case that $\pi_{1}(X-\Sigma)$ is trivial, it is straightforward to show that $\Sigma_{K}(\varphi)$ is topologically isotopic to $\Sigma$ using topological surgery. Recently, S. Finashin 4 and the first-named author [15] have used variations of rim surgery to find smoothly knotted surfaces whose complements have (nontrivial) cyclic fundamental groups. It is interesting to ask whether these surfaces are topologically nontrivial. Indeed, Finashin [4, page 50] asks about the topological classification of his surfaces, while the first author [15] showed that for many knots $K$ and choices of $\varphi$, the surface $\Sigma_{K}(\varphi)$ is topologically equivalent to the original $\Sigma$. In this paper, we show that any surface produced by a torus knot surgery whose complement has cyclic fundamental group is topologically standard.

To state the main result, we need to define the surgery operation in question.

Definition 1.1. Let $Z$ be a 4 -manifold containing a torus $T$ of self-intersection 0 , and let $K$ be a knot with exterior $E(K)$. Denote by $\mu_{T}$ the boundary of the normal disk of the torus, and let the longitude/meridian of $\mathrm{K}$ be $\lambda_{K}$ and $\mu_{K}$, respectively. Let $\varphi: \partial \nu(T) \rightarrow S^{1} \times \partial E(K)$ be any diffeomorphism such that $\varphi_{*} \mu_{T}=\lambda_{K}$.

(a) Knot surgery is the operation $Z \rightarrow Z_{K}(\varphi)=Z-\left(T \times D^{2}\right) \cup_{\varphi}\left(S^{1} \times E(K)\right)$.

Received by the editors October 4, 2006.

2000 Mathematics Subject Classification. Primary 57R57.

Key words and phrases. Rim surgery, knotted surface, surgery theory.

The second author was partially supported by NSF Grant 0505605. 
(b) If $\pi_{1} Z_{K}(\varphi) \cong \pi_{1} Z$ is cyclic, then the knot surgery will be called a cyclic surgery.

Note that the boundary of $Z$ is not touched by this operation, so it is meaningful to ask if there is a diffeomorphism or homeomorphism $Z_{K}(\varphi) \rightarrow Z$ extending the identity map on $\partial Z$. Fintushel and Stern [5] investigated this construction when $Z=X-\nu(\Sigma)$ is the exterior of an embedded surface in the closed manifold $X^{4}$. Gluing back in the neighborhood of the surface gives a new embedding of $\Sigma$ in $X_{K}(\varphi)$, with image $\Sigma_{K}(\varphi)$. Fintushel and Stern focused particularly on a torus $T$ that is the preimage in $\partial \nu(\Sigma)$ of a closed curve $\alpha \subset \Sigma$, which they called a rim torus. In this case (referred to as rim surgery) there is a canonical identification of $X$ and $X_{K}(\varphi)$, and we view $\Sigma_{K}(\varphi)$ as lying in $X$. If an appropriate SeibergWitten invariant of $X$ is nontrivial, and the Alexander polynomial of $K$ is not 1, then $\Sigma_{K}(\varphi)$ is smoothly knotted. On the other hand, if $\pi_{1}(X-\Sigma)=1$, then rim surgery does not change the topological type of the embedding. Our main result is a generalization of this last statement.

Theorem 1.2. Let $\Sigma \subset X$ be an embedded surface such that $\pi_{1}(X-\Sigma) \cong \mathbb{Z}_{d}$. Suppose that $T$ is a torus in the complement of $\Sigma$, and that the knot surgery $(X, \Sigma) \rightarrow\left(X_{K}(\varphi), \Sigma_{K}(\varphi)\right)$ is cyclic. Then there is a pairwise homeomorphism $\left(X_{K}(\varphi), \Sigma_{K}(\varphi)\right) \rightarrow(X, \Sigma)$.

The proof of the theorem uses the traditional paradigm of surgery theory: we will find a homotopy equivalence, modify it so that it is normally bordant to the identity, and then show that the normal bordism may be surgered to give an s-cobordism.

In certain cases, there is a canonical diffeomorphism between $X$ and $X_{K}(\varphi)$, and we regard $\Sigma$ and $\Sigma_{K}(\varphi)$ as embedded surfaces in $X$. The examples we have in mind are the rim surgery referred to previously, and the annulus rim surgery in Finashin's paper [4]. In this circumstance, it is reasonable to ask if $\Sigma$ and $\Sigma_{K}(\varphi)$ are isotopic.

Theorem 1.3. Suppose that $X$ is simply connected and $\pi_{1}(X-\Sigma)$ is cyclic. If $\Sigma_{K}(\varphi)$ is obtained from $\Sigma$ by a cyclic rim surgery or annulus rim surgery, then $\Sigma_{K}(\varphi)$ is topologically isotopic to $\Sigma$.

The final section of the paper contains some examples of cyclic surgeries to which Theorems 1.2 and 1.3 apply.

Remark 1.4. There are presumably alternate ways to obtain these results, most notably the general methods for topological surgery with finite fundamental group due to Hambleton and Kreck [13, 12, 11, that can perhaps be applied in this context. (Compare [3, 18.) We are able to use the more traditional surgery theory because we are able to construct a specific homotopy equivalence between $Z$ and $Z_{K}(\varphi)$, whose normal invariant can be readily calculated by geometric means.

\section{Proofs of THE THEOREMS}

We start with a simple way to recognize a homotopy equivalence between 4manifolds with finite $\pi_{1}$.

Lemma 2.1. Let $M^{\prime}$ and $M$ be oriented 4-manifolds with finite fundamental group. Suppose that $f:\left(M^{\prime}, \partial M^{\prime}\right) \rightarrow(M, \partial M)$ is a degree-1 map with the following properties. 
(1) $f: \partial M^{\prime} \rightarrow \partial M$ is a homotopy equivalence.

(2) $f_{*}: \pi_{1} M^{\prime} \stackrel{\partial}{\cong} \pi_{1} M$.

(3) $f_{*}: H_{*}\left(M^{\prime} ; \mathbb{Z}\right) \stackrel{\cong}{\cong} H_{*}(M ; \mathbb{Z})$.

Then $f$ is a homotopy equivalence.

Proof. Denote by $\tilde{M} \rightarrow M$ the universal cover, and let $d$ be its degree. By the Whitehead theorem (see 2] for the statement in the nonsimply connected case) it suffices to show that the lift $\tilde{f}$ of $f$ to the universal cover induces an isomorphism in integral homology. Since the degree of $f$ is 1 , the same is true for $\tilde{f}$, and so the induced map in homology is a surjection. Now $H_{1}\left(\tilde{M}^{\prime} ; \mathbb{Z}\right)=H_{1}(\tilde{M} ; \mathbb{Z})=0$, and Poincaré duality (plus the fact that $\tilde{f}$ is a homotopy equivalence on the boundary) shows that $f_{*}: H_{3}\left(\tilde{M}^{\prime} ; \mathbb{Z}\right) \stackrel{\cong}{\longrightarrow} H_{3}(\tilde{M} ; \mathbb{Z})$.

Again, the fact that the degree of $\tilde{f}$ is one implies that

$$
f_{*}: H_{2}\left(\tilde{M}^{\prime} ; \mathbb{Z}\right) \longrightarrow H_{2}(\tilde{M} ; \mathbb{Z}),
$$

a map between two free abelian groups, is a surjection. On the other hand, we know from (3) that the Euler characteristics of $M$ and $M^{\prime}$ are equal, and so $\chi\left(\tilde{M}^{\prime}\right)=$ $d \cdot \chi(M)=d \cdot \chi\left(M^{\prime}\right)=\chi(\tilde{M})$. It follows that $H_{2}\left(\tilde{M}^{\prime} ; \mathbb{Z}\right)$ and $H_{2}(\tilde{M} ; \mathbb{Z})$ have the same rank, and so $\tilde{f}_{*}$ must in fact be an isomorphism.

Let $X$ be a simply connected oriented 4-manifold. For an oriented embedded surface $\Sigma \subset X$, let $Z=X-\nu(\Sigma)$ be its exterior; note that $\pi_{1}(Z)$ is normally generated by the meridian of $\Sigma$, which we will denote by $\mu_{\Sigma}$. If $\pi_{1}(Z)$ is cyclic, then of course $\mu_{\Sigma}$ is a generator. Suppose that $T \subset Z$ is an embedded torus such that $T \cdot T=0$, with a framing of its normal bundle, giving an identification $\partial \nu(T) \cong T^{3}$. (In the case of rim surgery, the framing will be canonical.) Let $\left(S^{3}, K\right)$ be a knot with exterior $E(K)$. The choice of a diffeomorphism $\varphi: T^{3} \rightarrow S^{1} \times \partial E(K)$ then gives a manifold $Z_{K}(\varphi)=Z-\nu(T) \cup_{\varphi}\left(S^{1} \times E(K)\right)$.

The gluing map $\varphi$ can be encoded as a $3 \times 3$ matrix with respect to a basis $\left\{\alpha, \beta, \mu_{T}\right\}$ for $H_{1}\left(T^{3}\right)$ and $\left\{S^{1}, \mu_{K}, \lambda_{K}\right\}$ for $H_{1}\left(S^{1} \times \partial E(K)\right)$. We will always assume that $\varphi$ takes $\mu_{T}$ to $\lambda_{K}$, so the matrix will have the form

$$
\varphi=\left(\begin{array}{lll}
a & b & 0 \\
c & d & 0 \\
p & q & 1
\end{array}\right)
$$

where $a d-b c=1$.

If $a=d=1$ and $b=q=0$, then such a gluing corresponds, in the original setting of rim surgery, to replacing the spinning construction of Fintushel-Stern with a combination of ( $c$-fold) twist spinning [32] and ( $p$-fold) roll spinning [7, 20. The matrix formulation of these classical constructions from high-dimensional knot theory is described in great detail in [26] and [23, 22.

Lemma 2.2. Suppose that $\varphi$ is a gluing map with $\varphi\left(\mu_{T}\right)=\lambda_{K}$, and that $K$ is the unknot $O$. Then $Z_{O}(\varphi) \cong Z$ via a diffeomorphism that is the identity on the boundary.

Proof. Consider the following decompositions of $Z$ and $Z_{O}(\varphi)$ :

$$
Z=Z-\left(T \times D^{2}\right) \cup_{i d}\left(S^{1} \times E(O)\right)
$$


and

$$
Z_{O}(\varphi)=Z-\left(T \times D^{2}\right) \cup_{\varphi}\left(S^{1} \times E(O)\right) .
$$

Then it is sufficient to extend $i d \circ \varphi^{-1}: S^{1} \times \partial E(O) \rightarrow S^{1} \times \partial E(O)$ to a diffeomorphism $S^{1} \times E(O) \rightarrow S^{1} \times E(O)$. Using a diffeomorphism of $T^{3}$ that preserves the fiber $\mu_{T}$, we may assume that (2.1) has the simpler form

$$
\varphi=\left(\begin{array}{lll}
1 & 0 & 0 \\
0 & 1 & 0 \\
p & q & 1
\end{array}\right) .
$$

Considering $S^{1} \times E(O)$ as $S_{x}^{1} \times S_{y}^{1} \times D^{2}$, we can write an element in $S^{1} \times E(O)$ as $\left(e^{2 \pi x i}, e^{2 \pi y i}, r e^{2 \pi z i}\right)$. Then we define an extension of $\varphi^{-1}$ as follows;

$$
\varphi^{-1}\left(e^{2 \pi x i}, e^{2 \pi y i}, r e^{2 \pi z i}\right)=\left(e^{2 \pi x i}, e^{2 \pi y i}, r e^{2 \pi(z-r p x-r q y) i}\right),
$$

where $0 \leq x, y, z$, and $r \leq 1$.

Because of Lemma 2.2, it will suffice to show that for any cyclic surgery involving the $K$ there is a homeomorphism $Z_{K}(\varphi) \cong Z_{O}(\varphi)$.

Lemma 2.3. Let $K$ be a knot in $S^{3}$, and suppose that $Z \rightarrow Z_{K}(\varphi)$ is a surgery where $\pi_{1}\left(Z_{K}(\varphi)\right)$ is finite. There is an orientation preserving homotopy equivalence $f: Z_{K}(\varphi) \stackrel{\simeq}{\longrightarrow} Z_{O}(\varphi)$.

Proof. A straightforward obstruction theory argument produces a map $g: E(K) \rightarrow$ $E(O) \cong S^{1} \times D^{2}$ that induces an isomorphism in integral homology, and takes meridian to meridian, and longitude to longitude. Take the product of this map with the identity map on the circle, to get a homology equivalence $S^{1} \times E(K) \rightarrow$ $S^{1} \times E(O)$. Because we use the same gluing map $\varphi$ for both knots, this can be glued to the identity map on $Z-\nu(T)$ to get a map $f: Z_{K}(\varphi) \rightarrow Z_{O}(\varphi)$. The 5-lemma implies that $f$ induces an isomorphism on integral homology, and it is an isomorphism on $\pi_{1}$ as well. The result follows by Lemma 2.1.

We will refer to the homotopy equivalence provided by Lemma 2.3 as the canonical homotopy equivalence. It is automatically a normal map 31, and we would like to construct a normal cobordism from $f$ to the identity map of $Z_{O}(\varphi)$, which will be denoted $Z^{\prime}$ henceforth. Recall that a (topological) normal map $h: Y \rightarrow$ $Z^{\prime}=Z_{O}(\varphi)$, relative to the identity on the boundary, is classified by an element $n(h) \in\left[Z^{\prime}, \partial Z^{\prime} ; G / T O P\right]$. A standard calculation [17] says that this is isomorphic to $H^{4}\left(Z^{\prime}, \partial Z^{\prime}\right) \oplus H^{2}\left(Z^{\prime}, \partial Z^{\prime} ; \mathbb{Z}_{2}\right)$, and that the first component of $n(h)$ is just $\frac{1}{8}\left(\sigma(Y)-\sigma\left(Z^{\prime}\right)\right)$. In the case at hand, the signature of $Y=Z_{K}(\varphi)$ is the same as that of $Z^{\prime}$, so we concentrate on the second component, which we will denote by $S(f)$.

Note that the copy of $S^{1} \times E(O)$ used in constructing $Z^{\prime}$ contains a torus $T^{\prime}=$ $S^{1} \times \mu_{K}$. The homology class of this torus in $Z^{\prime}$ will be denoted $\left[T^{\prime}\right]$.

Lemma 2.4. For the canonical homotopy equivalence $f$, the invariant $S(f) \in$ $H^{2}\left(Z^{\prime}, \partial Z^{\prime} ; \mathbb{Z}_{2}\right)$ is given by $\operatorname{Arf}(K) \operatorname{PD}\left(T^{\prime}\right)$.

Proof. Since $H^{2}\left(Z^{\prime}, \partial Z^{\prime} ; \mathbb{Z}_{2}\right) \cong \operatorname{Hom}\left(H_{2}\left(Z^{\prime}, \partial Z^{\prime} ; \mathbb{Z}_{2}\right) ; \mathbb{Z}_{2}\right)$, the class $S(f)$ is determined by its evaluation on (possibly nonorientable) surfaces $(G, \partial G) \subset\left(Z^{\prime}, \partial Z^{\prime}\right)$. The following recipe for this evaluation is generally viewed as part of Sullivan's characteristic variety theorem [29, 30]. We could not find this exact statement in the 
literature, and so have supplied a proof in an appendix to this paper. Make $f$ transverse to $G$ to get a 2 -dimensional normal map $f^{-1} G \rightarrow G$. Then $\langle S(f),[G, \partial G]\rangle$ is the surgery obstruction of this normal map (in $L_{2}(1) \cong \mathbb{Z}_{2}$ ) which in turn is given by the Arf invariant. Note that this evaluation depends on the relative homology class $[G, \partial G]$, but not on the choice of surface representing that class.

Now given a surface $G \subset Z^{\prime}$, its mod-2 intersection number with $T^{\prime}$ is either 0 or 1 . By changing $G$ by a homology (in fact by an embedded cobordism) we may assume that the intersection of $G$ with $S^{1} \times E(O) \subset Z^{\prime}$ is, correspondingly, either empty or a single copy of the disk spanning $O$ in $E(O)$. This uses the fact that the boundary of this disk is preserved by the gluing map $\varphi$. If $G \cdot T^{\prime}=0$, so the intersection is empty, then $f$ is a homeomorphism on $f^{-1} G$, and so the Arf invariant computing $\langle S(f),[G, \partial G]\rangle$ must also vanish.

On the other hand, if $G \cdot T^{\prime}=1$, so the intersection is the meridional disk, then we can decompose $G=G_{0} \cup D^{2}$ where the $D^{2}$ is the disk bounding $O$ in $E(O)$. Clearly $f^{-1} G$ decomposes in a corresponding way, where $f: f^{-1} G_{0} \rightarrow G_{0}$ is a homeomorphism and $f^{-1} D^{2}$ is a Seifert surface for $K$, mapping with degree 1 onto $D^{2}$. Thus $\langle S(f),[G, \partial G]\rangle$ is the Arf invariant of a quadratic form defined on $\operatorname{ker}\left[H_{1}\left(f^{-1} G ; \mathbb{Z}_{2}\right) \rightarrow H_{1}\left(G ; \mathbb{Z}_{2}\right)\right]$. But this kernel is clearly the same as the $(\bmod 2)$ homology of the Seifert surface, and it is straightforward to identify the quadratic form with the one that gives the Arf invariant of $K$. Putting together these two cases, we get that $\langle S(f),[G, \partial G]\rangle=\operatorname{Arf}(K) G \cdot T^{\prime}=\operatorname{Arf}(K)\left\langle\operatorname{PD}\left(T^{\prime}\right),[G, \partial G]\right\rangle$.

From this lemma, if the Arf invariant of $K$ is trivial, then the homotopy equivalence constructed in Lemma 2.3 is normally cobordant to the identity. On the other hand, if $\operatorname{Arf}(K)=1$, then $g$ is not normally cobordant to the identity, and so this construction does not work. However, we will see that this Arf invariant can be 'absorbed into the complement' by choosing a different homotopy equivalence.

Proof of Theorem 1.2, We claim that there is a homotopy equivalence (relative to the boundary) $f^{\prime}: Z^{\prime} \rightarrow Z^{\prime}$ with the same normal invariant as $f$. If the Arf invariant of $K$ is trivial, then the normal invariant of $f$ is trivial, and so $f$ is normally cobordant to the identity. In fact, the normal cobordism is easy to construct 'by hand'. Following [8, 9, the vanishing of $\operatorname{Arf}(K)$ gives rise to an explicit normal cobordism, relative to the boundary, of the map $g$ to the identity of $E(O)$. As in the proof of Lemma 2.3, this normal cobordism (crossed with $S^{1}$ ) can be glued to the identity map on $S^{1} \times(Z-\nu(T))$ to give the desired normal cobordism.

If $\operatorname{Arf}(K)=1$, then we make use of a classic construction of Wall [31, §16] that is discussed carefully in [1, section 5]. Given a class in $\pi_{2}\left(Z^{\prime}\right)$ represented by a map $\alpha: S^{2} \rightarrow Z^{\prime}$, consider the composition $\alpha \circ \eta^{2} \in \pi_{4} Z^{\prime}$, where $\eta^{2}$ is the generator of $\pi_{4}\left(S^{2}\right)$. Then the following composition, say $f_{\alpha}$, is a homotopy equivalence (rel $\left.\partial Z^{\prime}\right)$ :

$$
Z^{\prime} \longrightarrow Z^{\prime} \vee S^{4} \stackrel{\alpha \circ \eta^{2}}{\longrightarrow} Z^{\prime}
$$

The normal invariant $S\left(f_{\alpha}\right)$ is computed geometrically in Theorem 5.1 of [1] and is given by

$$
\left(1+\left\langle w_{2}\left(Z^{\prime}\right), \alpha\right\rangle\right) \operatorname{PD}(\alpha)
$$

Here we have used the same notation for the homotopy class $\alpha$ and the homology class it carries. 
Note that the image of the Hurewicz map is determined via the Hopf exact sequence

$$
\pi_{2}(X) \stackrel{h}{\longrightarrow} H_{2}(X) \longrightarrow H_{2}\left(\pi_{1}(X)\right) \rightarrow 0 .
$$

Since $H_{2}\left(\mathbb{Z}_{d}\right)=0$, the Hurewicz map is onto, so in particular the class $T^{\prime}$ is spherical and we get a homotopy equivalence $f_{T^{\prime}}: Z^{\prime} \rightarrow Z^{\prime}$. Recall that $T^{\prime}=S^{1} \times \mu_{K} \subset$ $S^{1} \times E(K)$, so it has trivial normal bundle. Thus $\left\langle w_{2}\left(Z^{\prime}\right), T^{\prime}\right\rangle \equiv T^{\prime} \cdot T^{\prime} \equiv 0(\bmod 2)$, and so by Lemma 2.4 and formula (2.3), both $S\left(f_{T^{\prime}}\right)$ and $S(f)$ are given by $\operatorname{PD}\left(T^{\prime}\right)$. This establishes the claim.

A normal cobordism $W$ from $f: Z_{K}(\varphi) \rightarrow Z^{\prime}$ to the homotopy equivalence $f^{\prime}$ : $Z^{\prime} \rightarrow Z^{\prime}$ has a surgery obstruction that a priori lies in the Wall group $L_{5}^{h}\left(\mathbb{Z}\left[\mathbb{Z}_{d}\right]\right)$. But in fact [14, section 11] this group, and the group $L^{s}\left(\mathbb{Z}\left[\mathbb{Z}_{d}\right]\right)$ vanish, so that $W$ may be surgered to give an s-cobordism rel boundary between $Z_{K}(\varphi)$ and $Z^{\prime}$, which is topologically a product. Hence the identity map between $\partial Z_{K}(\varphi)$ and $\partial Z^{\prime}$ extends to a homeomorphism. (For $d$ odd and $Z$ closed, this argument can be found in [19].) Finally, since the map on the boundary is the identity, the homeomorphism extends over $X$.

Proof of Theorem 1.3. Note that when $T$ is a rim torus (or annular rim torus as in [4), the homology class $T$ is trivial in $H_{2}(X)$. So the homeomorphism constructed in the proof of Theorem 1.2 has trivial normal invariant when viewed as a map from $X$ to $X$. Hence by 27] (again see 11 for full details) that homeomorphism is homotopic to the identity. Then the work of Perron [25] and Quinn [27] says that this homeomorphism is isotopic to the identity. The isotopy takes $\Sigma$ onto $\Sigma_{K}(\varphi)$.

\section{EXAMPLES OF CYCLIC SURGERIES}

In this section, we discuss those examples we know of gluing maps which produce a surface whose complement has a cyclic fundamental group. In many cases, these will be smoothly knotted, but Theorem 1.3 shows that they are topologically standard. In all of these, $\Sigma$ is a surface in the simply connected manifold $X$ with $\pi_{1}(X-\Sigma) \cong \mathbb{Z} / d$.

3.1. Rim surgery ([5]). Let $T$ be a rim torus in $X-\Sigma$, i.e. of the form $\alpha \times \mu_{\Sigma}$ where $\alpha$ is a non-separating curve in $\Sigma$. The simplest gluing map

$$
\varphi: \partial \nu(T)\left(\cong T \times \partial D^{2}\right) \rightarrow S^{1} \times \partial E(K)
$$

sends $\alpha \mapsto\left[S^{1}\right], \mu_{\Sigma} \mapsto \mu_{K}$, and $\partial D^{2} \mapsto \lambda_{K}$. As shown in [15, Example 3.2], the fundamental group of $X-\Sigma_{K}(\varphi)$ contains the fundamental group of the $d$-fold branched cover of $\left(S^{3}, K\right)$, and hence (by a strong form of the Smith conjecture 24]) is bigger than $\mathbb{Z} / d$.

Cyclic surgeries arise when we perform twisting ( $m$ times) and rolling ( $n$ times) in the gluing map $\varphi$, which has the form

$$
\varphi=\left(\begin{array}{lll}
1 & 0 & 0 \\
m & 1 & 0 \\
n & 0 & 1
\end{array}\right) .
$$

The terminology (twisting and rolling) is explained in Section 2 In the case of $m$ twisted rim surgery (i.e. $n=0$ ) it is shown in [15] that for any knot $K, \pi_{1}\left(Z_{K}(\varphi)\right.$ ) is cyclic when $d \equiv \pm 1(\bmod m)$, but can also be noncyclic when this condition is 
violated. For instance, if $d \mid m$ and $K$ is nontrivial, then as in Example 3.2 of [15], the group $\pi_{1}\left(Z_{K}(\varphi)\right)$ will not be cyclic. We will show that $\varphi$ is a cyclic surgery for any knot $K$ in a more general case which includes an arbitrary amount of rolling.

Proposition 3.1. If $(d, m)=1$ and $n$ is arbitrary, then in the above language, the $m$-twisted $n$-rolled rim surgery has $\pi_{1}\left(Z_{K}(\varphi)\right)=\mathbb{Z}_{d}$ for any knot $K$.

To prove this, we will first describe $m$-twists and $n$-rolls of rim surgery following [15. Given a nonseparating curve $\alpha$ in $\Sigma$, choose a neighborhood of $\alpha$ in $X$ of the form $S^{1} \times I \times D^{2}=S^{1} \times B^{3}$, where $S^{1} \times I$ is a neighborhood of $\alpha$ in $\Sigma$. Adjusting a trivialization of the normal bundle $\nu(\Sigma \times I)$ in $X$, we may assume that the push off of the curve $\alpha$ along the trivialization is homologically trivial in $X-\Sigma$. Now we define self-diffeomorphisms denoted by $\tau$ and $\rho$ on $\left(S^{3}, K\right)$, as it follows. Define a twist, $\tau$, by

$$
\tau\left(\bar{\theta}, e^{i \varphi}, t\right)=\left(\bar{\theta}, e^{i(\varphi+2 \pi t)}, t\right) \quad \text { for } \quad\left(\bar{\theta}, e^{i \varphi}, t\right) \in K \times \partial D^{2} \times I,
$$

and otherwise, $\tau(y)=y$. (Here we use $K \cong S^{1} \cong \mathbb{R} / \mathbb{Z}$.) Similarly, a roll, $\rho$, is given by

$$
\rho\left(\bar{\theta}, e^{i \varphi}, t\right)=\left(\overline{\theta+t}, e^{i \varphi}, t\right) \quad \text { for } \quad\left(\bar{\theta}, e^{i \varphi}, t\right) \in K \times \partial D^{2} \times I,
$$

and otherwise, $\rho(y)=y$. It is useful to note that $\rho$ and $\tau$ commute.

Divide $\left(S^{3}, K\right)$ into two $\operatorname{arcs}\left(B^{3}, K_{+}\right) \cup\left(B_{-}^{3}, K_{-}\right)$, where the second pair is unknotted and $B_{-}^{3}$ lies inside the tubular neighborhood $K \times D^{2}$. The pair $\left(X, \Sigma_{K}(\varphi)\right)$ defined by $m$-twisted and $n$-rolled rim surgery is then obtained by taking out the neighborhood of $\alpha$ and gluing back the mapping torus of $\left(B^{3}, K_{+}\right)$with monodromy given by powers of the diffeomorphisms $\tau$ and $\rho$ :

$$
\left(X, \Sigma_{K}(\varphi)\right)=(X, \Sigma)-S^{1} \times\left(B^{3}, I\right) \cup_{\partial} S^{1} \times_{\rho^{n} \tau^{m}}\left(B^{3}, K_{+}\right) .
$$

As in 15, we observe that this description of $\left(X, \Sigma_{K}(\varphi)\right)$ is equivalent to the one obtained by performing knot surgery in Definition 1.1 along the rim torus $T$ and the given matrix $\varphi$ in (3.1).

Now let's consider $d$-fold covers of $X$ branched along $\Sigma$ and $\Sigma_{K}(\varphi)$ denoted by $Y$ and $Y_{K}(\varphi)$, respectively. From the decomposition of $\left(X, \Sigma_{K}(\varphi)\right)$ in (3.4) and the choice of the curve $\alpha$, we easily observe that $Y_{K}(\varphi)$ is obtained by doing a surgery on $Y$ as follows:

$$
\left(Y_{K}(\varphi), \Sigma_{K}(\varphi)\right)=(Y, \Sigma)-S^{1} \times\left(B^{3}, I\right) \cup_{\partial} S^{1} \times_{\tilde{\rho}^{n}} \tilde{\tau}^{m}\left(B^{3}, K_{+}\right)^{d},
$$

where $\left(B^{3}, K_{+}\right)^{d}$ is a $d$-fold cover of $B^{3}$ branched along $K_{+}$and $\tilde{\rho}, \tilde{\tau}$ are lifts of $\rho, \tau$, respectively. Here we consider $\left(B^{3}, K_{+}\right)^{d}$ as the punctured $d$-fold branched cover $\left(S^{3}, K\right)^{d}$ of $\left(S^{3}, K\right)$. The lift $\tilde{\rho}$ of $\rho$ into $\left(S^{3}, K\right)^{d}$ is a map defined by rolling along the lifted knot of $K$ on a collar of $\partial E(K)^{d}$ in a $d$-fold cover of the exterior $E(K)^{d}$ as described in (3.3), and otherwise, the identity. However $\tilde{\tau}$ is a little more complicated. Let $\sigma$ be the canonical covering translation of the group $\mathbb{Z}_{d}$ of covering transformations that is a rotation by $2 \pi / d$ about the branch set. Then the following map gives the lift of $\tilde{\tau}$ on $\left(S^{3}, K\right)^{d}$ :

$$
\tilde{\tau}= \begin{cases}\sigma(x) & \text { if } x \in E(K)^{d}-\partial E(K)^{d} \times I, \\ \left(\bar{\theta}, e^{i(s / d \cdot 2 \pi+\varphi)}, s\right) & \text { if } x=\left(\bar{\theta}, e^{i \varphi}, s\right) \in \partial E(K)^{d} \times I, \\ x & \text { otherwise. }\end{cases}
$$


Note that $\tilde{\rho}$ is isotopic to the identity on $\left(S^{3}, K\right)^{d}$. The isotopy $F_{t}$ between identity and $\tilde{\rho}^{-1}$ is defined on a collar of $\partial E(K)^{d}$ as follows:

$$
F_{t}\left(\bar{\theta}, e^{i \varphi}, s\right)=\left(\overline{\theta-t s}, e^{i \varphi}, s\right) \quad \text { for } \quad\left(\bar{\theta}, e^{i \varphi}, s\right) \in \partial E(K)^{d} \times I .
$$

This isotopy induces a homeomorphism $\left(S^{3}, K\right)^{d} \times_{\tilde{\rho}} S^{1} \rightarrow\left(S^{3}, K\right)^{d} \times S^{1}$. So we rewrite $Y_{K}(\varphi)$ with a gluing map $f$ along the boundary:

$$
(Y, \Sigma)-\left(B^{3}, I\right) \times S^{1} \cup_{f^{n}}\left(B^{3}, K_{+}\right)^{d} \times_{\tilde{\tau}^{m}} S^{1} .
$$

Here we need to make use of some results from Plotnick's paper [26. Consider a plumbing $P$, at two points, of two copies of $S^{2} \times D^{2}$. The pair of cores of the $S^{2} \times D^{2}$ s is called a 'twin' by Montesinos 223, 22, note that $\partial P$ is a 3 -torus. Plotnick 26] constructed infinitely many homotopy spheres by gluing to $S^{1} \times E(K)$ using an identification $A$ defined on the boundary of $P$. More explicitly, write

$$
\Omega_{A}=P \cup_{A} S^{1} \times E(K),
$$

where $A$ is expressed by a matrix form according to a certain basis $\left\{e_{1}, e_{2}, e_{3}\right\}$ on $H_{1}(\partial P)$ and each $e_{i}$ represents a curve on $\partial P$. (For details, see [26].) The general form of $A$ for which $\Omega_{A}$ is a homology sphere is

$$
\left(\begin{array}{ccc}
p & k & 0 \\
-\gamma & \beta & 0 \\
-\alpha \gamma+b p & \alpha \beta+b k & 1
\end{array}\right) .
$$

In many cases, this construction produces $S^{4}$. In $\Omega_{A}$, we get a knot $A(K)$ determined by one of the two cores of $P$; in the description in [26] it is the second of the spheres. An interesting result is that the knot $A(K)$ in $\Omega_{A}$ is fibered. Moreover, in some cases the fiber is described explicitly in terms of branched covers [26, Theorem 5.6].

Proof of Proposition 3.1. Consider $\left(B^{3}, K_{+}\right)^{d} \times_{\tilde{\tau} m} S^{1}$ in the decomposition (3.7) of $Y_{K}(\varphi)$. Then there is an interesting connection with Plotnick's construction [26]. To see this, consider our assumption $(d, m)=1$. So there are $\gamma$ and $\beta$ such that $d \gamma+m \beta=1$. If we do Plotnick's construction using the matrix

$$
A=\left(\begin{array}{ccc}
m & d & 0 \\
-\gamma & \beta & 0 \\
0 & 0 & 1
\end{array}\right),
$$

then the resulting space $\Omega_{A}$ is the $m$-fold cyclic branched cover of the $d$-twist spin of $K$ [32. Theorem 5.6 in [26] says that the knot $A(K)$ produced in $\Omega_{A}$ is fibered, and its fiber is exactly $\left(B^{3}, K_{+}\right)^{d}$. Moreover its characteristic map is the same as $\tilde{\tau}^{m}$ described in (3.6). In other words, $\left(B^{3}, K_{+}\right)^{d} \times_{\tilde{\tau}^{m}} S^{1}$ is the complement of $A(K)$ in $\Omega_{A}$.

Comparing this gluing map $A$ (3.9) with the general form (3.8), in our circumstance we have $p=m, k=d,-\alpha \gamma+b m=0$ and $\alpha \beta+b d=0$. Since $d \gamma+m \beta=1$, we have $d \gamma b+m \beta b=b$. Since $b m=\alpha \gamma$ and $b d=-\alpha \beta,-\alpha \beta \gamma+\alpha \beta \gamma=b$. So $b=0$. This implies that $\alpha=0$. According to Corollary 6.1 in [26], this means that $\Omega_{A}$ is smoothly $S^{4}$. Thus, $\left(B^{3}, K_{+}\right)^{d} \times \tilde{\tau}^{m} S^{1}$ is smoothly $S^{4}-A(K)$. Then $Y_{K}(\varphi)$ is isomorphic to

$$
(Y, \Sigma)-\left(B^{3}, I\right) \times S^{1} \cup_{f^{n}} S^{4}-A(K) .
$$

This implies that $Y$ and $Y_{K}(\varphi)$ are homeomorphic. 
Now we consider $d$-fold unbranched covers of $Z=X-\Sigma$ and $Z_{K}(\varphi)=X-\Sigma_{K}(\varphi)$ denoted by $\widetilde{Z}$ and $\widehat{Z_{K}(\varphi)}$, respectively. Considering the description (3.5) of the branched cover $Y_{K}(\varphi)$, we can similarly write the unbranched cover $\widehat{Z_{K}(\varphi)}$ as

$$
\widetilde{Z}-S^{1} \times\left(B^{3}, I\right) \cup_{\partial} S^{1} \times_{\tilde{\rho}^{n} \tilde{\tau}^{m}} E(K)^{d} .
$$

Let's apply the Van Kampen Theorem to this decomposition. Since $\widetilde{Z}-S^{1} \times$ $\left(B^{3}, I\right)$ is homotopy equivalent to $\widetilde{Z}$ and $\pi_{1} \widetilde{Z}=\{1\}$, the fundamental group $\pi_{1}\left(\widehat{Z_{K}(\varphi)}\right)$ is isomorphic to $\pi_{1}\left(S^{1} \times \tilde{\rho}^{n} \tilde{\tau}^{m} E(K)^{d}\right) / \operatorname{im} j$ where

$$
j: \pi_{1}\left(S^{1} \times\left(S^{2}-\{2 p t s\}\right)\right) \rightarrow \pi_{1}\left(S^{1} \times \tilde{\rho}^{n} \tilde{\tau}^{m} E(K)^{d}\right)
$$

is the inclusion homomorphism. Eventually, we have

$$
\pi_{1}\left(\widehat{Z_{K}(\varphi)}\right) \cong\left\langle\pi_{1}\left(E(K)^{d}, *\right) \mid \mu_{\tilde{K}}=1, \beta=\tilde{\rho}_{*}^{n} \tilde{\tau}_{*}^{m}(\beta), \forall \beta \in \pi_{1}\left(E(K)^{d}, *\right)\right\rangle
$$

where $\mu_{\tilde{K}}$ is a meridian of the lifted knot $K$. To assert $\pi_{1}\left(Z_{K}(\varphi)\right)=\mathbb{Z}_{d}$, it is sufficient to show that $\pi_{1}\left(\widehat{Z_{K}(\varphi)}\right)$ is trivial. First, note that $\pi_{1}\left(Y_{K}(\varphi)\right)$ is trivial. Applying the Van Kampen Theorem to the decomposition for $Y_{K}(\varphi)$ in (3.5), we rewrite the group $\pi_{1}\left(Y_{K}(\varphi)\right)$ as

$$
\left\langle\pi_{1}\left(\left(B^{3}, K_{+}\right)^{d}, *\right) \mid \beta=\tilde{\rho}_{*}^{n} \tilde{\tau}_{*}^{m}(\beta), \forall \beta \in \pi_{1}\left(\left(B^{3}, K_{+}\right)^{d}, *\right)\right\rangle .
$$

Since $\pi_{1}\left(\left(B^{3}, K_{+}\right)^{d}, *\right) \cong \pi_{1}\left(E(K)^{d}, *\right) /\left\langle\mu_{\tilde{K}}\right\rangle$, this implies that the generator and relations in (3.10) make $\pi_{1}\left(\widetilde{Z_{K}(\varphi)}\right)$ trivial as well.

Remark 3.2. We were led to the statement of Proposition 3.1 by some computations with the group theory program GAP [10]. We investigated $m$-twisted, $n$-rolled rim surgery for some simple knots. We found, for instance, that if $K$ is a figure 8 knot, then 1-twisted, $n$-rolled rim surgery is a cyclic surgery for $n \leq 100$ (independent of $d$ ). However, the computations did not finish in a reasonable amount of time for more complicated knots and larger values of $m$ or $n$. In retrospect, it seems remarkable that Plotnick was able to show that in many cases $\Omega_{A}$ is actually $S^{4}$, whereas showing that its fundamental group is trivial seems somewhat difficult, computationally.

3.2. Finashin's construction. Let's briefly review the annulus rim surgery construction from [4. Suppose that there is a smoothly embedded surface $M$ in $X$, called a 'membrane', such that $M \cong S^{1} \times I, M \cap \Sigma=\partial M$ and $M$ meets $\Sigma$ normally along $\partial M$. By adjusting a trivialization of its regular neighborhood $U$, we can assume that $U\left(\cong S^{1} \times B^{3}\right) \cap \Sigma=S^{1} \times f$, where $f=I_{0} \sqcup I_{1}=I \times \partial I$ is a disjoint union of two unknotted segments of a part of the boundary of a band $b=I \times I$ in $B^{3}$. Here the band $b=I \times I$ is trivially embedded in $B^{3}$ and the intersection $I \times I \cap \partial B^{3}=\partial I \times I$. Then given a knot $K$ in $S^{3}$, we consider a band $b_{K} \subset B^{3}$ obtained by knotting $b$ along $K$, and we denote by $f_{K}$ the pair of arcs bounding $b_{K}$. Note that the framing of $b_{K}$ is chosen the same as the framing of $b$ (see [4, Fig. 1]). Then we get a new surface $\Sigma_{K}$ as follows:

$$
\left(X, \Sigma_{K}\right)=(X, \Sigma)-S^{1} \times\left(B^{3}, f\right) \cup S^{1} \times\left(B^{3}, f_{K}\right) .
$$

There is an equivalent description for this construction using knot surgery. Let $\gamma$ be a meridian of $b$ in $B^{3}$. Then there is a torus $T \subset \nu(M)$ corresponding to $S^{1} \times \gamma \subset S^{1} \times\left(B^{3}, f\right)$, and $\Sigma_{K}$ is constructed by knot surgery on this torus. In Finashin's original construction, the gluing map $\varphi$ sends $\left[S^{1}\right] \mapsto\left[S^{1}\right], \gamma \mapsto \mu_{K}$, and 
$\partial D^{2} \mapsto \lambda_{K}$. More generally, we can do $m$-twists and $n$-rolls in this construction, resulting in a gluing map $\varphi$ of (3.1).

Proposition 3.3. Knot surgery using $\varphi$ from (3.1) preserves the fundamental group $\pi_{1}\left(Z_{K}(\varphi)\right)=\mathbb{Z}_{d}$. In other words, $m$-twisted and $n$-rolled annulus rim surgery is a cyclic surgery.

Proof. Let's consider the process of $m$-times twist-spinning and $n$-times rolling of a knot $K$ in $S^{3}$. Choose a regular neighborhood $\nu(K)$ containing a knotted band $b_{K}$ described in Finashin's construction. We define self-diffeomorphisms $\tau$ and $\rho$ on $\left(S^{3}, \nu(K)\right)$ in the same way as (3.2) and (3.3). Then $\tau$ is fixed on $\partial E(K)$ and with support in a collar of $\partial E(K)$, and it induces a conjugation by a meridian. Similarly, $\rho$ is a conjugation by a longitude. Then we can observe that $Z_{K}(\varphi)$ obtained by knot surgery (Definition 1.1) along the above torus $T$ is equivalent to

$$
Z-S^{1} \times\left(B^{3}, f\right) \cup S^{1} \times \rho^{n} \tau^{m}\left(B^{3}-f_{K}\right) .
$$

Applying the Van Kampen theorem to this decomposition of $Z_{K}(\varphi)$ and following the similar argument in [4], we can get that

$$
\pi_{1}\left(Z_{K}(\varphi)\right) \cong \pi_{1}\left(S^{1} \times \rho^{n} \tau^{m}\left(B^{3}-f_{K}\right)\right) / j(k),
$$

where $j: \pi_{1}\left(S^{1} \times \partial\left(B^{3}-f\right) \cong S^{1} \times\left(S^{2}-\{4 p t s\}\right)\right) \rightarrow \pi_{1}\left(S^{1} \times \rho_{\rho^{n} \tau^{m}}\left(B^{3}-f_{K}\right)\right)$ is the inclusion homomorphism and $k$ is the kernel of the inclusion homomorphism $\pi_{1}\left(S^{1} \times \partial\left(B^{3}-f\right)\right) \rightarrow \pi_{1}\left(Z-S^{1} \times\left(B^{3}, f\right)\right)$. The group $\pi_{1}\left(S^{1} \times \partial\left(B^{3}-f\right) \cong\right.$ $\left.S^{1} \times\left(S^{2}-\{4 p t s\}\right)\right)$ is generated by $\left[S^{1}\right]$ and three generators denoted by $a_{1}, a_{2}$, and $a_{3}$ for $S^{2}-\{4 p t s\}$, where $i\left(a_{1}\right)=i\left(a_{2}\right)$ is represented by a loop around one arc of $f$ in $B^{3}$ and $i\left(a_{3}\right)$ is represented by a loop around $f$ under the inclusion $i: \pi_{1}\left(\partial\left(B^{3}-f\right)\right) \rightarrow \pi_{1}\left(B^{3}-f\right)$. (See Figure 1.) So the kernel $k$ is generated by

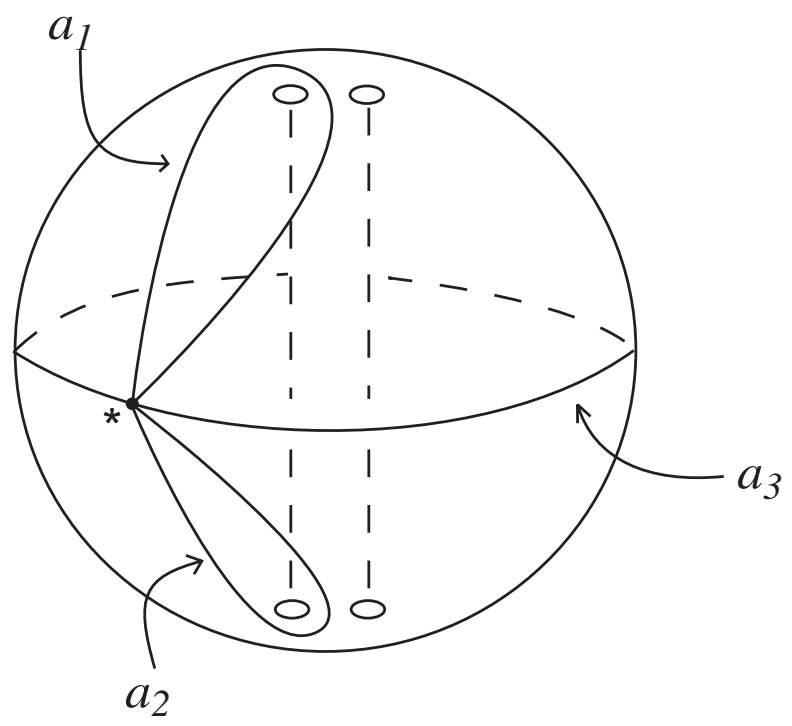

FiguRE 1 
$\left[S^{1}\right], a_{1}^{d}, a_{1} a_{2}^{ \pm 1}$, and $a_{3}$. Since $j\left(\left[S^{1}\right]\right)$ kills the first factor of $\pi_{1}\left(S^{1} \times \rho^{n} \tau^{m}\left(B^{3}-f_{K}\right)\right)$, the group $\pi_{1}\left(Z_{K}(\varphi)\right)$ can be written by

$$
\left\langle\pi_{1}\left(B^{3}-f_{K}, *\right) \mid a_{1}^{d}=1, \beta=\rho_{*}^{n} \tau_{*}^{m}(\beta), a_{3}=1, a_{1} a_{2}^{ \pm 1}=1, \forall \beta \in \pi_{1}\left(B^{3}-f_{K}, *\right)\right\rangle
$$

where we omit $j$ to simplify the notation. As discussed in 4, the relation $a_{3}$ has the same effect on $\pi_{1}\left(B^{3}-f_{K}, *\right)$ as attaching a 2-cell along a loop $m_{b}$ turning around $b_{K}$ in $B^{3}$. Adding this 2-cell makes $f_{K}$ an unknotted arc $I$ in $B^{3}$. (See Figure 2 which is the same figure in [4].) So only one generator $a_{1}$ of $\pi_{1}\left(\partial\left(B^{3}-f\right)\right)$
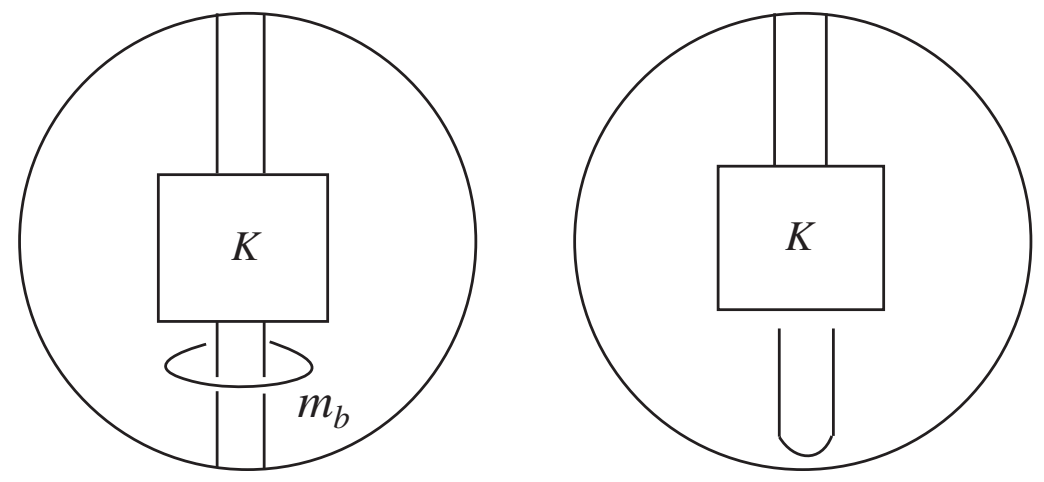

FiguRE 2

becomes the generator for $\pi_{1}\left(B^{3}-I\right)$ (to see this, note that $a_{1}$ is represented by a loop around $I)$. By geometric observation, we know that $\tau_{*}\left(a_{1}\right)$ is the conjugate of $a_{1}$ by $a_{3}$ and $\rho_{*}\left(a_{1}\right)$ is the conjugate of $a_{1}$ by a longitude $\lambda_{K}$. But since $a_{3}$ and the longitude are killed in the group $\pi_{1}\left(B^{3}-I\right)$, the factorization by $j(k)$ makes the group $\pi_{1}\left(Z_{K}(\varphi)\right)$ into a cyclic group $\mathbb{Z}_{d}$.

\section{Appendix A. Normal maps in Dimension 4}

In the proof of Lemma 2.4, we gave a formula for evaluating the normal invariant of a map of 4-manifolds. It was pointed out to us by Ian Hambleton that Sullivan's characteristic variety theorem, on which this evaluation is based, does not have a complete proof in the literature. Because 4 is a fairly low dimension, it is possible to assemble a straightforward proof based on well-established facts in surgery theory, and we present such a proof in this appendix.

We treat the closed PL case first; extension to the bounded case is routine. The TOP case follows by the same reasoning using the computation [16] of the homotopy groups of $G / T O P$. The statement we want is that PL normal maps to a closed oriented manifold $X$ are determined by two invariants, $S(f)$ and $\sigma(f)$, where $\sigma$ is the simply connected surgery obstruction, given by the difference in signature divided by 8 . By the Universal Coefficient Theorem, $S(f) \in H^{2}(X ; Z / 2)$ is determined by the pairings $\langle S(f), S\rangle$ for $S$ a surface (not necessarily orientable) in $X$. The claim is that (for $f$ transverse to $S$ ) this evaluation is the Arf invariant of the normal map $f^{-1}(S) \rightarrow S$.

The proof starts from the fact, proved by transversality and valid in any dimension, that PL normal maps to a closed manifold $X$ are given by homotopy 
classes $[X, G / P L]$. Now it is well-known (cf. for example [21, §4]) that the first two nonzero homotopy groups of $G / P L$ are $\pi_{2}=\mathbb{Z} / 2$ and $\pi_{4}=\mathbb{Z}$, and $\pi_{5}=0$. The first $k$-invariant in the PL case is $\delta S q^{2}$; in the TOP case it is trivial. This is enough to determine that $[X, G / P L]$ is the set $\left\{(a, s) \in H^{2}(X ; \mathbb{Z} / 2) \oplus H^{4}(X ; \mathbb{Z}) \mid a^{2}=s\right.$ $(\bmod 2)\}$.

Suppose that $F: M \rightarrow X$ is a normal map, and that $S \subset X$ is an embedded surface with $F$ transverse to $S$. Then the restriction of $F$ is a normal map $f$ : $F^{-1}(S) \rightarrow S$. Moreover, the classifying map for $f=$ normal map of surfaces in $[S, G / P L]$ is the composition of the classifying map for $F$ in $[X, G / P L]$ with the inclusion $i: S \rightarrow X$. This holds because the normal bundle of $F^{-1}(S)$ in $M$ is the pull-back of the normal bundle of $S$ in $X$.

The formula we need then follows from the following statement: if $f: S^{\prime} \rightarrow$ $S$ is a normal map of surfaces classified by a map $g: S \rightarrow G / P L$, and $a \in$ $H^{2}(G / P L ; \mathbb{Z} / 2)=\mathbb{Z} / 2$ is the generator, then $g^{*}(a)=\operatorname{Arf}(f)$. This can be seen in many ways, e.g. it follows directly from [28, Theorem 4.1].

\section{ACKNOWLEDGMENTS}

We thank Ian Hambleton for some helpful correspondence on surgery theory, and for urging us to include a proof of our assertions about the normal invariant.

\section{REFERENCES}

[1] Tim D. Cochran and Nathan Habegger, On the homotopy theory of simply connected four manifolds, Topology 29 (1990), no. 4, 419-440. MR.1071367 (91h:57006)

[2] James F. Davis and Paul Kirk, Lecture notes in algebraic topology, Graduate Studies in Mathematics, vol. 35, American Mathematical Society, Providence, RI, 2001. MR1841974 (2002f:55001)

[3] S. M. Finashin, M. Kreck, and O. Ya. Viro, Nondiffeomorphic but homeomorphic knottings of surfaces in the 4-sphere, Topology and geometry-Rohlin Seminar, Lecture Notes in Math., vol. 1346, Springer, Berlin, 1988, pp. 157-198. MR970078 (90h:57021)

[4] Sergey Finashin, Knotting of algebraic curves in $\mathbb{C P}^{2}$, Topology 41 (2002), no. 1, 47-55. MR:1871240 (2003c:57042)

[5] Ronald Fintushel and Ronald J. Stern, Surfaces in 4-manifolds, Math. Res. Lett. 4 (1997), no. 6, 907-914. MR.1492129 (98k:57047)

[6] _ Knots, links, and 4-manifolds, Invent. Math. 134 (1998), no. 2, 363-400. MR99j:57033

[7] R. H. Fox, Rolling, Bull. Amer. Math. Soc. 72 (1966), 162-164. MR0184221 (32:1694)

[8] Michael H. Freedman, A surgery sequence in dimension four; the relations with knot concordance, Invent. Math. 68 (1982), no. 2, 195-226. MR666159 (84e:57006)

[9] Michael H. Freedman and Frank Quinn, Topology of 4-manifolds, Princeton Mathematical Series, vol. 39, Princeton University Press, Princeton, NJ, 1990. MR.1201584 (94b:57021)

[10] The GAP Group, GAP - Groups, Algorithms, and Programming, Version 4.4, 2006 (http://www.gap-system.org).

[11] Ian Hambleton and Matthias Kreck, On the classification of topological 4-manifolds with finite fundamental group, Math. Ann. 280 (1988), no. 1, 85-104. MR928299 (89g:57020)

[12] _ Cancellation, elliptic surfaces and the topology of certain four-manifolds, J. Reine Angew. Math. 444 (1993), 79-100. MR1241794 (95h:57036)

[13] _ Cancellation of hyperbolic forms and topological four-manifolds, J. Reine Angew. Math. 443 (1993), 21-47. MR1241127 (94k:57030)

[14] Ian Hambleton and Laurence R. Taylor, A guide to the calculation of the surgery obstruction groups for finite groups, Surveys on surgery theory, Vol. 1, Ann. of Math. Stud., vol. 145, Princeton Univ. Press, Princeton, NJ, 2000, URL:http://www.math.mcmaster.ca/ian/published/Lgroups_2000.pdf, $\quad$ pp. 225-274. MR.1747537 (2001e:19007) 
[15] Hee Jung Kim, Modifying surfaces in 4-manifolds by twist spinning, Geom. Topol. 10 (2006), 27-56 (electronic). MR2207789

[16] Robion C. Kirby and Laurence C. Siebenmann, "Foundational essays on topological manifolds, smoothings, and triangulations", Princeton University Press, Princeton, N.J., 1977. With notes by John Milnor and Michael Atiyah, Annals of Mathematics Studies, No. 88.

[17] Robion C. Kirby and Laurence R. Taylor, A survey of 4-manifolds through the eyes of surgery, Surveys on surgery theory, Vol. 2 (S. Cappell, A. Ranicki, and J. Rosenberg, eds.), Ann. of Math. Stud., vol. 149, Princeton Univ. Press, Princeton, NJ, 2001, pp. 387-421. MR2002a:57028

[18] Matthias Kreck, On the homeomorphism classification of smooth knotted surfaces in the 4-sphere, Geometry of low-dimensional manifolds, 1 (Durham, 1989), London Math. Soc. Lecture Note Ser., vol. 150, Cambridge Univ. Press, Cambridge, 1990, pp. 63-72. MR1171891 (93k:57042)

[19] Terry C. Lawson, Inertial h-cobordisms with finite cyclic fundamental group, Proc. Amer. Math. Soc. 44 (1974), 492-496. MR0358820 (50:11279)

[20] R. A. Litherland, Deforming twist-spun knots, Trans. Amer. Math. Soc. 250 (1979), 311-331. MR530058 (80i:57015)

[21] I. Madsen and R. J. Milgram, "The classifying spaces for surgery and cobordism of manifolds", vol. 92 of Annals of Mathematics Studies, Princeton University Press, Princeton, N.J., 1979. MR.548575 (81b:57014)

[22] José M. Montesinos, On twins in the four-sphere. I, Quart. J. Math. Oxford Ser. (2) 34 (1983), no. 134, 171-199. MR698205 (86i:57025a)

[23] José M. Montesinos, On twins in the four-sphere. II, Quart. J. Math. Oxford Ser. (2) 35 (1984), no. 137, 73-83. MR734666 (86i:57025b)

[24] John W. Morgan and Hyman Bass (eds.), The Smith conjecture, Pure and Applied Mathematics, vol. 112, Academic Press, Inc., Orlando, FL, 1984. Papers presented at the symposium held at Columbia University, New York, 1979. MR758459 (86i:57002)

[25] Bernard Perron, Pseudo-isotopies et isotopies en dimension quatre dans la catégorie topologique, Topology 25 (1986), no. 4, 381-397. MR.862426 (89g:57024)

[26] Steven P. Plotnick, Fibered knots in $S^{4}$-twisting, spinning, rolling, surgery, and branching, Four-manifold theory (Durham, N.H., 1982), Contemp. Math., vol. 35, Amer. Math. Soc., Providence, RI, 1984, pp. 437-459. MR.780592 (87a:57021)

[27] Frank Quinn, Isotopy of 4-manifolds, J. Differential Geom. 24 (1986), no. 3, 343-372. MR868975 (88f:57020)

[28] Colin P. Rourke and Dennis P. Sullivan, On the Kervaire obstruction, Ann. of Math. (2), 94 (1971), 397-413. MR0305416 (46:4546)

[29] Dennis P. Sullivan, Geometric topology: localization, periodicity and Galois symmetry, KMonographs in Mathematics, vol. 8, Springer, Dordrecht, 2005, The 1970 MIT notes, edited and with a preface by Andrew Ranicki. MR2162361

[30] Dennis P. Sullivan, Triangulating and smoothing homotopy equivalences and homeomorphisms. Geometric Topology Seminar Notes, in "The Hauptvermutung book", vol. 1 of $K$ Monogr. Math., Kluwer Acad. Publ., Dordrecht, 1996, 69-103. MR1434103 (98c:57027)

[31] C. T. C. Wall, Surgery on compact manifolds, Academic Press, London, 1970, London Mathematical Society Monographs, No. 1. MR0431216 (55:4217)

[32] E. C. Zeeman, Twisting spun knots, Trans. Amer. Math. Soc. 115 (1965), 471-495. MR0195085 (33:3290)

Department of Mathematics, Louisiana State University, Baton Rouge, Louisiana 70803-4918

E-mail address: heekim@lsu.edu

Department of Mathematics, MS 050, Brandeis University, Waltham, Massachusetts 02454

E-mail address: ruberman@brandeis.edu 